\title{
Comparison of Pre-service Teachers' Social Skill Levels
}

\author{
Ercüment Erdoğan*, Berati Çargıt
}

School of Physical Education and Sports, Ordu University, Ordu, Turkey

Copyright $(\mathrm{C} 2018$ by authors, all rights reserved. Authors agree that this article remains permanently open access under the terms of the Creative Commons Attribution License 4.0 International License

\begin{abstract}
The aim of this study was to compare the social skill levels of university students from three different teaching departments. Study sample consisted of 100 students (mean age $=21.55 \pm 0.165$ ) of physical education and sports teaching (PEST) department, 100 students (mean age $=20.93 \pm 0.157)$ of classroom teaching $(\mathrm{CT})$ department and 100 students (mean age $=21.43 \pm 0.189$ ) of music teaching (MT) department. Data were collected using a social skills inventory and a socio-demographic information form. Descriptive statistics, one-way variance analysis, LSD multiple comparison test and independent sample t test were used to analyze the data using the SPSS. The mean scores of PEST, CT and MT students on the Social Skills Inventory were $280.81( \pm 4.56), 281.34( \pm 5.45)$ and $278.94( \pm 5.32)$, respectively. There was no statistically significant difference in social skills scores between PEST, CT and MT students while female participants' social skills scores were significantly higher than those of male participants. Social skills scores did not differ significantly by parents' education level while they differed significantly by place of residence (village, district or city). Participants have an average level of social skills with no departmental difference. Female pre-service teachers have a higher level of social skills than male pre-service teachers.
\end{abstract}

Keywords Social Skills Level, Pre-service Teacher, University Student

\section{Introduction}

Social skills are important behavioral elements used to initiate and maintain positive interactions with other people. Yüksel [1] defines social skills as learnable, socially acceptable, influential, target-specific, contextual and communication-facilitating behaviors involving observable and unobservable cognitive and affective items that bring about positive reactions and prevent negative reactions. Sorias [2] defines social skills as learned behaviors that enable us to interact with others successfully, express our feelings, defend personal rights, ask for help from others when necessary and refuse requests that are in conflict with our beliefs, ideals and priorities. Social skills are learned first in the family and then in school. Teachers, therefore, play a critical role in the development of social and communication skills in students. However, it is also of significance that teachers have socially acceptable behaviors that enable interaction with others and the ability to assist and encourage their students to develop these skills. If the teacher is capable of paying attention and responding to students' physical and psychological needs, then students can bond with him or her. The establishment of this bond can help students engage in their own development [3]. Although there are many studies on students' social skills, the number of studies on teachers' social skills is very limited. Yet, social skills are learned through observation and imitation, and reinforced by positive feedback [4]. Capel et al. classifies behaviors expected from teachers into three categories: 1organization, planning and teaching approach, 2professionalism and 3- social skills [5]. According to Giblin [6], learning to establish positive relationships with other people makes up $85 \%$ of a successful career and $99 \%$ of personal happiness.

Good education depends especially on good communication between teachers and students [7]. Almost all research shows that teacher effectiveness is closely related to student success and satisfaction [8]. Sports enable people to participate in dynamic social circles and therefore plays a key role in socialization and development of social skills. Given that sports are often collective activities in modern societies, people interested in sports can engage in social relationships with different groups of people through sporting activities. Sports enable people to look out beyond their own narrow circles and to be in dialogue with other people with different beliefs and thoughts, to influence and be influenced by them. Sports, therefore, enable new friendships and social cohesion [9]. Music requires understanding of others as well as describing events, objects and facts. It can also be a means, purpose and method in the development of emotions and social relations [10]. Music teachers with good communication skills enable students to better express themselves in musical terms and to display their skills freely. 
In this respect, determining the level of social skills of university students from different teaching departments will help those students to improve themselves and to be ready and prepared for their future role as teachers. This study aimed to investigate the social skill levels of university students from different departments. The aim of this study is to compare the social skill levels of pre-service teachers from different branches of universities based on different variables.

\section{Materials and Methods}

\subsection{Sampling}

The study sample consisted of 100 students of physical education and sports teaching (PEST) department, 100 students of classroom teaching (CT) department and 100 students of music teaching (MT) department of Ordu University.

\subsection{Data Collection}

Developed by Riggio in 1986 [4] and revised to its current form in 1989, the Social Skills Inventory (SSI) is a self-defining test consisting of 90 items. The SSI includes six subscales that measure social communication skills at two levels: emotional (nonverbal communication) and social (verbal communication). The SSI assesses expressive (encoding), sensitivity (decoding) and control (regulatory) skills. Expressive, sensitivity and control skills refer to one's ability to send messages, to interpret received messages and to manage the communicative process in various social situations, respectively. The subscales of the SSI are: emotional expressivity (EE), emotional sensitivity (ES), emotional control (EC), social expressivity (SE), social sensitivity (SS) and social control (SC). The total score ranges from 90 to 450 . The SSI was adapted to Turkish language, and its validity and reliability were established by Yüksel [7]. Riggio found the reliability coefficient of the SSI as $\mathrm{r}=.94$, while the reliability coefficient of the subscales ranged from $\mathrm{r}=.81$ to $\mathrm{r}=.96$. Yüksel [7] found the reliability coefficient of the SSI as $r$ $=.92$. Based on the possible range of scores from 90 to 450, social skills scores between 90 and 252 are categorized as low, those between 253 and 311 as average and those between 312 and 450 as high [8]. While the reliability coefficient of the subscales ranged from $\mathrm{r}=.81$ to $\mathrm{r}=.96$. Yüksel [7] found the reliability coefficient of the SSI as $r$ $=.92$. Based on the possible range of scores from 90 to 450, social skills scores between 90 and 252 are categorized as low, those between 253 and 311 as average and those between 312 and 450 as high [8].

\subsection{Personal Information Form}

The personal information form developed by the researcher consists of items pertaining to participants' demographic characteristics such as age, gender, education level of mother, education level of father and place of residence

\subsection{Data Analysis}

Data were analyzed using the Statistical Package for Social Sciences (SPSS), version 22. Social skills inventory total score and subscale scores were calculated using the calculation method of the inventory itself. Descriptive statistics, one-way ANOVA, LSD multiple comparison test and independent sample $t$ test were used to determine whether there was a statistically significant difference in scores between variables at a significance level of 0.05 .

\section{Findings}

Variance analysis results show that participants' total SSI, and EE, ES, EC, SE, SS and SC scores are 0.689, $0.114,0.207,0.511,0.734,0.911$ and 0.989 , respectively, indicating that there is no statistically significant difference in scores between departments $(\mathrm{P}>0.05)$ (Table 2). Pre-service physical education teachers have higher EE, EC and SS mean scores than other pre-service teachers. Pre-service classroom teachers have the highest ES, SE and TOTAL SSI mean scores. Pre-service classroom teachers have the highest mean score on social skill levels while pre-service music teachers have the lowest.

According to the t-test results, there is a statistically significant difference in EE $(p=0.049)$, ES ( $p=0.00)$, SE $(p=0.047)$ and SS $(p=0.013)$ subscale scores between male and female participants $(\mathrm{p}<0.05)$ while there is no statistically significant difference in EC $(p=0.40)$ and SC $(p=0.750)$ scores between them $(p>0.05)$ (Table 3$)$. $)$. Female pre-service teachers have higher mean scores on all subscales than male ones, which means that female pre-service teachers are better than male ones, especially in terms of social and emotional sensitivity scale and total social skill level. These results indicate that female pre-service teachers pay attention to social norms, adapt to the environment, are good listeners and viewers, and can accurately and fully resolve the emotional states of other people.

Table 1. Descriptive statistics.

\begin{tabular}{cccccc}
\hline & & $\mathrm{N}$ & Mean \pm S.dev & Min & Max \\
\hline \multirow{4}{*}{ Age } & PEST & 100 & $2.55 \pm 0.165$ & 19 & 25 \\
\cline { 2 - 6 } & MT & 100 & $21.43 \pm 0.189$ & 19 & 26 \\
\cline { 2 - 6 } & CT & 100 & $20.93 \pm 0.157$ & 18 & 24 \\
\hline
\end{tabular}


Table 2. Variance Analysis Results of Social Skills Scores by Departments

\begin{tabular}{|c|c|c|c|c|c|}
\hline Varia & & $\mathrm{N}$ & & $\mathrm{f}$ & $\mathrm{p}$ \\
\hline \multirow{3}{*}{$\mathrm{EE}$} & PEST & 100 & $46.05 \pm 4.79$ & \multirow{3}{*}{2.185} & \multirow{3}{*}{114} \\
\hline & MT & 100 & $44.65 \pm 4.43$ & & \\
\hline & $\mathrm{CT}$ & 100 & $45.31 \pm 4.90$ & & \\
\hline \multirow{3}{*}{ ES } & PEST & 100 & $49.77 \pm 5.77$ & \multirow{3}{*}{1.585} & \multirow{3}{*}{207} \\
\hline & MT & 100 & $49.83 \pm 6.77$ & & \\
\hline & $\mathrm{CT}$ & 100 & $51.16 \pm 6.03$ & & \\
\hline \multirow{3}{*}{$\mathrm{EC}$} & PEST & 100 & $46.35 \pm 4.60$ & \multirow{3}{*}{673} & \multirow{3}{*}{511} \\
\hline & MT & 100 & $45.50 \pm 5.34$ & & \\
\hline & $\mathrm{CT}$ & 100 & $46.07 \pm 5.75$ & & \\
\hline \multirow{3}{*}{ SE } & PEST & 100 & $46.56 \pm 5.31$ & \multirow{3}{*}{310} & \multirow{3}{*}{734} \\
\hline & MT & 100 & $47.11 \pm 6.11$ & & \\
\hline & CT & 100 & $47.12 \pm 5.79$ & & \\
\hline \multirow{3}{*}{ SS } & PEST & 100 & $46.16 \pm 5.64$ & \multirow{3}{*}{094} & \multirow{3}{*}{911} \\
\hline & MT & 100 & $45.89 \pm 5.53$ & & \\
\hline & $\mathrm{CT}$ & 100 & $45.85 \pm 5.39$ & & \\
\hline \multirow{3}{*}{$\mathrm{SC}$} & PEST & 100 & $45.90 \pm 5.50$ & \multirow{3}{*}{011} & \multirow{3}{*}{989} \\
\hline & MT & 100 & $45.93 \pm 4.73$ & & \\
\hline & $\mathrm{CT}$ & 100 & $45.83 \pm 4.66$ & & \\
\hline \multirow{3}{*}{ Total SSI } & PEST & 100 & $280.81 \pm 20.34$ & \multirow{3}{*}{373} & \multirow{3}{*}{689} \\
\hline & MT & 100 & $278.94 \pm 21.50$ & & \\
\hline & $\mathrm{CT}$ & 100 & $281.34 \pm 20.44$ & & \\
\hline
\end{tabular}

Table 3. Analysis Results of Social Skills Scores by Gender

\begin{tabular}{|c|c|c|c|c|c|}
\hline Variable & Gender & $\mathrm{N}$ & $\begin{array}{c}\text { Mean } \pm \text { Std.de } \\
\text { v. }\end{array}$ & $\mathrm{t}$ & $\mathrm{p}$ \\
\hline \multirow{2}{*}{$\mathrm{EE}$} & Male & 142 & $44.73 \pm 4.23$ & \multirow{2}{*}{-1.974} & \multirow{2}{*}{$049^{\prime}$} \\
\hline & Female & 158 & $45.83 \pm 5.17$ & & \\
\hline \multirow{2}{*}{ ES } & Male & 142 & $48.44 \pm 6.10$ & \multirow{2}{*}{-4.755} & \multirow{2}{*}{$000^{*}$} \\
\hline & Female & 158 & $51.83 \pm 6.05$ & & \\
\hline \multirow{2}{*}{$\mathrm{EC}$} & Male & 142 & $45.73 \pm 4.42$ & \multirow{2}{*}{-.841} & \multirow{2}{*}{401} \\
\hline & Female & 158 & $46.25 \pm 5.92$ & & \\
\hline \multirow{2}{*}{ SE } & Male & 142 & $46.10 \pm 5.34$ & \multirow{2}{*}{-1.998} & \multirow{2}{*}{$047^{*}$} \\
\hline & Female & 158 & $47.43 \pm 6.02$ & & \\
\hline \multirow{2}{*}{ SS } & Male & 142 & $45.03 \pm 5.70$ & \multirow{2}{*}{-2.503} & \multirow{2}{*}{$013^{*}$} \\
\hline & Female & 158 & $46.64 \pm 5.29$ & & \\
\hline \multirow{2}{*}{$\mathrm{SC}$} & Male & 142 & $45.76 \pm 4.72$ & \multirow{2}{*}{-.319} & \multirow{2}{*}{750} \\
\hline & Female & 158 & $45.94 \pm 5.27$ & & \\
\hline \multirow{2}{*}{ Total SSI } & Male & 142 & $275.82 \pm 18.89$ & \multirow{2}{*}{-3.380} & \multirow{2}{*}{$001^{*}$} \\
\hline & Female & 158 & $283.96 \pm 21.94$ & & \\
\hline
\end{tabular}

${ }^{*} \mathrm{P}<0.05$

Participants' social skills scores did not differ significantly by fathers' education level $(p>0.05)$ (Table 4).
Table 4. Analysis Results of Social Skills Scores by Education Level of Father

\begin{tabular}{|c|c|c|c|c|c|}
\hline Variable & & $\mathrm{N}$ & Mean \pm Std.dev. & $\mathrm{f}$ & $\mathrm{P}$ \\
\hline \multirow{4}{*}{$\mathrm{EE}$} & İlliterate & 17 & $43.82 \pm 3.26$ & \multirow{4}{*}{869} & \multirow{4}{*}{483} \\
\hline & $\begin{array}{l}\text { Primary } \\
\text { School }\end{array}$ & 147 & $45.47 \pm 4.38$ & & \\
\hline & High School & 94 & $45.62 \pm 5.36$ & & \\
\hline & $\begin{array}{l}\text { Bachelor } \\
\text { degree }\end{array}$ & 42 & $44.85 \pm 4.93$ & & \\
\hline \multirow{4}{*}{ ES } & İlliterate & 17 & $45.33 \pm 4.73$ & \multirow{4}{*}{1.905} & \multirow{4}{*}{110} \\
\hline & $\begin{array}{l}\text { Primary } \\
\text { School }\end{array}$ & 147 & $46.76 \pm 6.19$ & & \\
\hline & High School & 94 & $50.53 \pm 6.36$ & & \\
\hline & $\begin{array}{l}\begin{array}{l}\text { Bachelor } \\
\text { degree }\end{array} \\
\end{array}$ & 42 & $50.47 \pm 6.36$ & & \\
\hline \multirow{4}{*}{$\mathrm{EC}$} & İlliterate & 17 & $50.41 \pm 5.04$ & \multirow{4}{*}{414} & \multirow{4}{*}{799} \\
\hline & $\begin{array}{l}\text { Primary } \\
\text { School }\end{array}$ & 147 & $50.67 \pm 6.22$ & & \\
\hline & High School & 94 & $45.76 \pm 4.32$ & & \\
\hline & $\begin{array}{l}\text { Bachelor } \\
\text { degree }\end{array}$ & 42 & $45.79 \pm 5.96$ & & \\
\hline \multirow{4}{*}{ SE } & $\underline{\text { Illiterate }}$ & 17 & $45.93 \pm 4.24$ & \multirow{4}{*}{1791} & \multirow{4}{*}{131} \\
\hline & $\begin{array}{l}\text { Primary } \\
\text { School }\end{array}$ & 147 & $46.85 \pm 5.09$ & & \\
\hline & High School & 94 & $45.97 \pm 5.25$ & & \\
\hline & $\begin{array}{l}\text { Bachelor } \\
\text { degree }\end{array}$ & 42 & $45.76 \pm 5.08$ & & \\
\hline \multirow{4}{*}{ SS } & $\underline{\text { Illiterate }}$ & 17 & $47.57 \pm 5.38$ & \multirow{4}{*}{1817} & \multirow{4}{*}{126} \\
\hline & $\begin{array}{l}\text { Primary } \\
\text { School }\end{array}$ & 147 & $46.42 \pm 6.02$ & & \\
\hline & High School & 94 & $46.56 \pm 6.34$ & & \\
\hline & $\begin{array}{l}\begin{array}{l}\text { Bachelor } \\
\text { degree }\end{array} \\
\end{array}$ & 42 & $46.93 \pm 5.74$ & & \\
\hline \multirow{4}{*}{$\mathrm{SC}$} & İlliterate & 17 & $43.52 \pm 5.90$ & \multirow{4}{*}{1069} & \multirow{4}{*}{372} \\
\hline & $\begin{array}{l}\text { Primary } \\
\text { School }\end{array}$ & 147 & $46.60 \pm 4.64$ & & \\
\hline & High School & 94 & $45.28 \pm 5.65$ & & \\
\hline & $\begin{array}{l}\begin{array}{l}\text { Bachelor } \\
\text { degree }\end{array} \\
\end{array}$ & 42 & $46.31 \pm 7.36$ & & \\
\hline
\end{tabular}

Participants' ES $(p=0.016)$ and SE $(p=0.01)$ scores significantly differed by mothers' education level $(p<$ $0.05)$ while their EE $(p=0.81)$, EC $(p=0.64), \operatorname{SS}(p=$ $0.90)$ and SC $(p=0.42)$ scores did not $(p>0.05)$ (Table 5). 
Table 5. Analysis Results of Social Skills Scores by Education Level of Mother

\begin{tabular}{|c|c|c|c|c|c|}
\hline \multicolumn{2}{|c|}{ Değişken } & \multirow{2}{*}{$\begin{array}{l}\mathrm{N} \\
40 \\
\end{array}$} & \multirow{2}{*}{$\begin{array}{l}\text { Mean } \pm \text { std.d } \\
44.82 \pm 4.54 \\
\end{array}$} & $\mathrm{f}$ & Anlamlılık \\
\hline \multirow{4}{*}{ DA } & Illiterate & & & \multirow{4}{*}{.394} & \multirow{4}{*}{.813} \\
\hline & $\begin{array}{c}\text { Primary } \\
\text { School } \\
\end{array}$ & 188 & $45.36 \pm 4.91$ & & \\
\hline & $\begin{array}{c}\text { High } \\
\text { School }\end{array}$ & 55 & $45.50 \pm 4.28$ & & \\
\hline & $\begin{array}{c}\text { Bachelor } \\
\text { degree }\end{array}$ & 17 & $45.87 \pm 4.88$ & & \\
\hline \multirow{4}{*}{$\mathrm{DD}$} & İlliterate & 40 & $45.33 \pm 4.73$ & \multirow{4}{*}{3.109} & \multirow{4}{*}{$.016^{*}$} \\
\hline & $\begin{array}{c}\text { Primary } \\
\text { School } \\
\end{array}$ & 188 & $49.72 \pm 5.18$ & & \\
\hline & $\begin{array}{c}\text { High } \\
\text { School } \\
\end{array}$ & 55 & $51.10 \pm 6.61$ & & \\
\hline & $\begin{array}{c}\text { Bachelor } \\
\text { degree }\end{array}$ & 17 & $48.72 \pm 5.53$ & & \\
\hline \multirow{4}{*}{ DK } & İlliterate & 40 & $47.43 \pm 3.96$ & \multirow{4}{*}{.629} & \multirow{4}{*}{.642} \\
\hline & $\begin{array}{c}\text { Primary } \\
\text { School } \\
\end{array}$ & 188 & $50.25 \pm 6.22$ & & \\
\hline & $\begin{array}{c}\text { High } \\
\text { School } \\
\end{array}$ & 55 & $45.85 \pm 4.95$ & & \\
\hline & $\begin{array}{c}\text { Bachelor } \\
\text { degree }\end{array}$ & 17 & $46.29 \pm 5.57$ & & \\
\hline \multirow{4}{*}{ SA } & İlliterate & 40 & $45.16 \pm 4.28$ & \multirow{4}{*}{.4809} & \multirow{4}{*}{$.001^{*}$} \\
\hline & $\begin{array}{c}\text { Primary } \\
\text { School } \\
\end{array}$ & 188 & $45.43 \pm 5.34$ & & \\
\hline & $\begin{array}{c}\text { High } \\
\text { School }\end{array}$ & 55 & $45.97 \pm 5.25$ & & \\
\hline & $\begin{array}{c}\text { Bachelor } \\
\text { degree }\end{array}$ & 17 & $45.37 \pm 4.89$ & & \\
\hline \multirow{4}{*}{$\mathrm{SD}$} & İlliterate & 40 & $47.93 \pm 5.88$ & \multirow{4}{*}{.254} & \multirow{4}{*}{.907} \\
\hline & $\begin{array}{c}\text { Primary } \\
\text { School } \\
\end{array}$ & 188 & $45.56 \pm 5.16$ & & \\
\hline & $\begin{array}{c}\text { High } \\
\text { School }\end{array}$ & 55 & $44.50 \pm 5.17$ & & \\
\hline & $\begin{array}{c}\text { Bachelor } \\
\text { degree }\end{array}$ & 17 & $46.93 \pm 5.74$ & & \\
\hline \multirow{4}{*}{ SK } & İlliterate & 40 & $45.97 \pm 4.70$ & \multirow{4}{*}{.966} & \multirow{4}{*}{.427} \\
\hline & $\begin{array}{c}\text { Primary } \\
\text { School } \\
\end{array}$ & 188 & $45.95 \pm 5.28$ & & \\
\hline & $\begin{array}{c}\text { High } \\
\text { School } \\
\end{array}$ & 55 & $45.72 \pm 5.11$ & & \\
\hline & $\begin{array}{c}\text { Bachelor } \\
\text { degree }\end{array}$ & 17 & $47.06 \pm 10.1$ & & \\
\hline
\end{tabular}

${ }^{*} \mathrm{P}<0.05$

According to the LSD test results, there is a statistically significant difference in ES subscale scores between participants whose mothers have a primary school degree and those whose mothers have a high school degree $(\mathrm{p}=$ 0.012 ), and between those whose mothers have a primary school degree and those whose mothers have a bachelor's degree $(p=0.011)$. There is a statistically significant difference in SE subscale scores between participants whose mothers have a primary school degree and those whose mothers are illiterate $(p=0.009)$, and between those whose mothers have a high school degree and those whose mothers have a bachelor's degree $(p=0.006)(p<$ 0.05 ). The results show that participants whose mothers have a high level of education have better social skills than those whose mothers have a low level of education, indicating that the former are better at sensing the emotional states of other people and acting accordingly, and using social and verbal speech communication skills in practice than the latter (Table 6).

Table 6. LSD Test Results of Social Skills Scores by Education Level of Mother

\begin{tabular}{|c|c|c|c|c|c|}
\hline Variable & 1 & $\mathrm{j}$ & $\begin{array}{c}\text { Mean } \\
\text { differencek }(1-\mathrm{j})\end{array}$ & Std. err. & $\mathrm{p}$ \\
\hline ES & $\begin{array}{l}\text { Primary } \\
\text { school }\end{array}$ & $\begin{array}{c}\text { İlliterate } \\
\text { High school } \\
\text { Bachelor } \\
\text { degree }\end{array}$ & $\begin{array}{l}1.37606 \\
2.37379 \\
3.98342\end{array}$ & $\begin{array}{c}1.06925 \\
.94138 \\
1.55523\end{array}$ & $\begin{array}{l}.199 \\
.012^{*} \\
.011^{*}\end{array}$ \\
\hline $\mathrm{SE}$ & $\begin{array}{c}\text { Primary } \\
\text { school }\end{array}$ & $\begin{array}{c}\text { İlliterate } \\
\text { High school } \\
\text { Bachelor } \\
\text { degree }\end{array}$ & $\begin{array}{l}2.56117 \\
2.37253 \\
3.93617\end{array}$ & $\begin{array}{c}.97738 \\
.86049 \\
1.42160 \\
\end{array}$ & $\begin{array}{l}.009^{*} \\
.006^{*} \\
.006^{*}\end{array}$ \\
\hline
\end{tabular}

$* \mathrm{P}<0.05$

According to the variance analysis results, participants' ES $(p=0.014)$ and $\mathrm{SC}(\mathrm{p}=0.037)$ subscale scores differed significantly by place of residence $(\mathrm{p}<0.05)$ while their EE $(p=0.190), E C(p=0.159), \operatorname{SE}(p=0.276)$ and SS $(p=0.937)$ subscale scores did not $(p>0.05)$ (Table 7).

Table 7. Analysis Results of Social Skills Scores by Place of Residence

\begin{tabular}{|c|c|c|c|c|c|}
\hline & iable & $\mathrm{N}$ & Mean \pm Std.dev. & $\mathrm{f}$ & $\mathrm{p}$ \\
\hline \multirow{3}{*}{$\mathrm{EE}$} & City & 154 & $45.02 \pm 4.46$ & \multirow{3}{*}{1.688} & \multirow{3}{*}{.190} \\
\hline & Town & 129 & $45.85 \pm 5.10$ & & \\
\hline & Village & 17 & $44.11 \pm 3.91$ & & \\
\hline \multirow{3}{*}{ ES } & City & 154 & $50.57 \pm 5.24$ & \multirow{3}{*}{4.320} & \multirow{3}{*}{.014} \\
\hline & Town & 129 & $50.43 \pm 7.06$ & & \\
\hline & Village & 17 & $46.00 \pm 6.44$ & & \\
\hline \multirow{3}{*}{$\mathrm{EC}$} & City & 154 & $46.53 \pm 5.21$ & \multirow{3}{*}{1.849} & \multirow{3}{*}{.159} \\
\hline & Town & 129 & $45.39 \pm 5.15$ & & \\
\hline & Village & 17 & $45.23 \pm 5.96$ & & \\
\hline \multirow{3}{*}{ SA } & City & 154 & $47.06 \pm 6.03$ & \multirow{3}{*}{1.291} & \multirow{3}{*}{.276} \\
\hline & Town & 129 & $47.06 \pm 5.46$ & & \\
\hline & Village & 17 & $44.76 \pm 4.91$ & & \\
\hline \multirow{3}{*}{ SS } & City & 154 & $46.03 \pm 5.42$ & \multirow{3}{*}{.065} & \multirow{3}{*}{.937} \\
\hline & Town & 129 & $45.94 \pm 5.60$ & & \\
\hline & Village & 17 & $45.52 \pm 5.33$ & & \\
\hline \multirow{3}{*}{$\mathrm{SC}$} & City & 154 & $45.45 \pm 4.59$ & \multirow{3}{*}{3.321} & \multirow{3}{*}{$.037^{*}$} \\
\hline & Town & 129 & $46.65 \pm 4.59$ & & \\
\hline & Village & 17 & $44.05 \pm 5.35$ & & \\
\hline
\end{tabular}

${ }^{*} \mathrm{P}<0.05$

The mean ES subscale score of participants living in villages is statistically significantly lower than that of participants living in districts $(\mathrm{p}=0.006)$ and cities $(\mathrm{p}=$ 0.004). The mean SC subscale score of participants living in districts is statistically significantly higher than those of participants living in villages $(p=0.042)$ and cities $(p=$ 0.043) $(\mathrm{P}<0.05)$. The results indicate that participants 
living in villages have more difficulty understanding other people's emotional states and are more reserved in social interactions (Table 8).

Table 8. Post-hoc Test Results of Subscale Scores by Place of Residence

\begin{tabular}{cccccc}
\hline \multirow{2}{*}{ Variable } & \multirow{2}{*}{1} & $\mathrm{j}$ & $\begin{array}{c}\text { Mean } \\
\text { diffence }\end{array}$ & Std.Dev. & $\mathrm{p}$ \\
\hline \multirow{2}{*}{ ES } & Town & City & -4.57792 & 1.57470 & $.004^{*}$ \\
\cline { 2 - 6 } & & Town & -4.43411 & 1.58980 & $.006^{*}$ \\
\hline \multirow{2}{*}{ SC } & Town & City & 1.19662 & .58751 & $.043^{*}$ \\
\cline { 2 - 6 } & & Village & 2.59234 & 1.27010 & $.042^{*}$ \\
\hline
\end{tabular}

${ }^{*} \mathrm{P}<0.05$

\section{Discussion and Conclusions}

The Social Skills Inventory (SSI) was applied to 300 students from PEST, CT and MT departments of Ordu University. Participants' total SSI score and subscale mean scores were calculated using Riggio and Carney's [9] scoring technique. The results show that participants have an average level of social skills. These results are similar to those reported by Avşar and Kuter [10], Avşar [11], Akpınar et al. [12], Dalkıran et al. [13], Şenol and Türkçapar [14] and Gezer et al. [15].

In this study, participants' social skills scores did not differ by department. Girgin et al. [16] reported statistically significant differences in EE, EC, SS and SC subscale scores among students from different departments. Şenol and Türkçapar [14] conducted a study on pre-service teachers from PEST and CT departments, and reported a statistically significant difference in ES, EC, SE and SS subscale scores between the two departments. Özcep and Mirzeoğlu [17] conducted a study on physical education and sports and classroom teachers' social skills, and reported a statistically significant difference in favor of the former. Avşar and Kuter [10] found no meaningful relationship between university students' departments and social skills scores. Although this result is similar to our finding, some of the results of this study are not consistent with those of previous studies. Research on social skills mostly focus on preschool and primary school students. Although the number of studies on teachers and pre-service teachers is limited, their results vary significantly. Therefore, further research is warranted to better understand the factors contributing to the development of teachers' and pre-service teachers' social skills.

Girgin et al. [16] compared men's and women's social skills, and found that men's EC subscale scores were significantly higher than those of women. Şenol and Türkçapar [14] reported that female pre-service teachers' ES subscale scores were significantly higher than those of male pre-service teachers while the EC subscale scores of the latter were significantly higher than those of the former. Aktı [18], Dicle [19], Kalafat [20], Jamyang-Tshering [21], Kazdin (1985) [22], Raine, (1993)
[23] and Avşar and Kuter [10] argue that gender has a significant effect on social skills scores and that women have a higher level of social skills than men.

The results of this study show that female participants have significantly higher total score and subscale scores (except for EC and SC subscales) than male participants. These results are consistent with the literature. We can therefore conclude that male participants have better control-related social skills while female participants have better expressivity- and sensitivity-related social skills. However, Avşar [11], Tekin et al. (2006) [24] and Özcep and Mirzeoğlu [17] came to the conclusion that gender has no significant effect on social skills.

There is also a statistically significant in ES and SE subscale scores between participants whose mothers have a primary school degree and those whose mothers have a high school degree or bachelor's degree. Girgin et al. [16] report that pre-service teachers whose mothers have a bachelor's degree have higher SE subscale scores than those whose mothers have a primary school degree while Avşar and Kuter [10] and Erdoğan et al. [25] report no relationship between students' social skills scores and their mothers' education level. The difference in results reported by these studies warrant further research on this topic.

The results of this study show no evidence for an effect of fathers' education level on participants' social skills scores, which is similar to the findings reported by Girgin et al. [16], Avşar and Kuter [10] and Erdoğan et al. [25]. Therefore, whether parents' education level has an effect on students' social skills levels is a moot point.

Participants' ES and SC subscale scores significantly differed by place of residence. The mean ES and SC subscale scores of participants living in villages were significantly lower than those of participants living in districts and cities. Avşar and Kuter [10] also reported that students living in villages had lower ES and SC subscale scores than those living in districts and cities.

All in all, the results show that participants have an average level of social skills with no departmental difference and that female pre-service teachers have a higher level of social skills than male pre-service teachers. The literature contains conflicting results concerning the effect of parents' education level on students' social skills levels. The results also suggest that place of residence has an effect on students' social skills levels, indicating that those living in villages have lower control-related social skills than those living in districts and cities. Teachers should develop social skills to be able to improve their students' knowledge and skills, and to communicate emotionally and socially with them in a healthy way in the process of education. For successful education, teachers should be able to send messages through effective use of body language, analyze students' messages accurately and quickly, communicate easily with students through oral communication, and receive and analyze students' 
feedback quickly and correctly. Successful education depends on the effective use of communication skills by teachers and students. Based on the results of this study, it is recommended that lesson plans focusing on the development of social skills be included in the curricula of higher education institutions for teachers to be successful in their profession.

\section{REFERENCES}

[1] Yüksel, G. (1997) "Sosyal Beceri Eitiminin Üniversite Örencilerinin Sosyal Beceri Düzeyine Etkisi”.

[2] Sorias, O. (1986) "Sosyal Beceriler ve Değerlendirme Yöntemleri", Psikoloji Dergisi, 5, s: 25-29.

[3] Jordan , D.W. (1997)"Social Skills Through Cooperative Learning", Educational Research ,p: 21

[4] Riggio, R. E. (1986). Assessment of basic social skills. Journal of Personality and social Psychology, 51(3), 649.

[5] Capel, S., Leask, M., \& Turner, T. (2006). Learning to teach in the secondary school: A companion to school experience. Routledge.

[6] Giblin, Robert L, MARRANE MTCHELL HANSON. (1995). Introduction to counseling and guidanca. Fourt Edition. Merrill An Imprint Of Prenticehallenglewoodcliffs, New Jersey Columbus, Ohio.

[7] Bolat, S. (1996). Eğitim örgütlerinde iletişim: Hacettepe Üniversitesi Eğitim Fakültesi uygulamas1. Hacettepe Eğitim Fakültesi Dergisi, 12, 75-80.

[8] Şen, H. S. ve Erişen, Y.(2002). Öğretmen yetiştiren kurumlarda öğretim elemanlarının etkili öğretmenlik özellikleri. Gazi Üniversitesi Gazi Eğitim Fakültesi Dergisi, 22(1), 99-116

[9] Çaha, Ö. (2000). Spora Yaslanarak Bir Nefes Alma, Ankara: Beta Yayınevi.

[10] Demirsöz, E. S., Kocabaş, A. (2008). Sınıf Öğretmenliği Öğretmen Adaylarının Müzik Başarılarıyla Duygusal Zekaları Arasındaki İlişki. Milli Eğitim, 180.

[11] Yüksel, G. (1998). Sosyal Beceri Envanterinin Türkçeye Uyarlanması: Geçerlilik Ve Güvenirlilik Çalışması" Türk Psikolojik Danışma Ve Rehberlik Dergisi, Ankara. Cilt: 3, Say1: 9, Ss : 37-46

[12] Yüksel, G. "Sosyal Beceri Envanteri El Kitabı." Ankara: Asil Yayın Dağıtım (2004).

[13] Riggio, R. E., \& Carney, D. C. (1989). Manual for the social skills inventory. Research ed. Consulting Psychologists. Pr., Palo Alto, CA.

[14] Avşar, Z., \& Kuter, F. Ö. (2007). Beden eğitimi ve spor bölümü öğrencilerinin sosyal beceri düzeylerinin belirlenmesi (Uludağ Üniversitesi örneği). Eğitimde Kuram ve Uygulama, 3(2), 197-206.

[15] Avşar, Z. (2004). Beden eğitimi ve spor öğretmenlerinin sosyal beceri düzeylerinin Belirlenmesi. Uludağ Üniversitesi Eğitim Fakültesi Dergisi, 17(2), 111-130.

[16] Akpinar, S., Akpinar, Ö., Nas, K., Temel, V., \& Birol, S. S.
(2015). Beden Egitimi ve Spor Yüksekokulunda Okuyan Öğrencilerinin Bazı Değişkenler Açısından Sosyal Beceri Düzeylerinin İncelenmesi, Sosyal ve Ekonomik Araştırmalar Dergisi, 17(28), 80.

[17] Dalkıran, O., Aslan, C. S., Gezer, E. D., \& Vardar, T. (2015). Sporcu Ve Sedanter Ögrencilerin Sosyal Beceri Düzeylerinin Karşılaştırılması-The Comparison Of Social Skill Level Of Sportsman And Sedantery Students. Mehmet Akif Ersoy Üniversitesi Sosyal Bilimler Enstitüsü Dergisi, 1(13), 224-230.

[18] Şenol, E., Türkçapar, Ü. (2016). Üniversite öğrencilerinin sosyal beceri düzeylerinin incelenmesi. Mehmet Akif Ersoy Üniversitesi Eğitim Fakültesi Dergisi, 1(40)

[19] Gezer, E. D., Aslan, C. S., Dalkıran, O., \& K1lc1gil, E.(2017). Comparison Of Social Skill Levels Of Elite Sportsmen At Different Sports Branches Accordıng To Selected Variables. Mehmet Akif Ersoy Üniversitesi Sosyal Bilimler Enstitüsü Dergisi, 9(22), 251-258.

[20] Girgin, G., Çetingöz, D., Vural, D. E. (2011). Öğretmen adaylarının sosyal beceri düzeylerinin incelenmesi. Kuramsal Eğitimbilim Dergisi, 4(1), 38-49.

[21] Özcep, M. C., Mirzeoğlu, D. (2007) İlköğretimde Görev Yapan Beden Eğitimi Ve Sınıf Öğretmenlerinin Sosyal Beceri Düzeylerinin Karşılaştırılması. Gazi Beden Eğitimi Ve Spor Bilimleri Dergisi, 12(4), 21-34.

[22] Aktı, S. 2011. İlköğretim sekizinci sınıf öğrencilerinin medya okuryazarlığı ile sosyal beceri düzeyleri arasındaki iliş̧inin belirlenmesi. Furat Üniversitesi Sosyal Bilimler Enstitüsü, Yayınlanmamış Yüksek Lisans Tezi, Elazığ.

[23] Dicle A. N. (2006) Üniversite öğrencilerinin sosyal beceri düzeylerinin duygusal zeka düzeyleri ve bazı kişisel özelliklerine göre incelenmesi, Yayımlanmamış Yüksek Lisans Tezi, On Dokuz Mayıs Üniversitesi, Sosyal Bilimler Enstitüsü, Samsun

[24] Kalafat, T. (2007) Üniversite öğrencilerinin beden memnuniyeti düzeyleri ile soysal beceri düzeyleri arasındaki ilişkinin karşılaştırmalı olarak incelenmesi (Çanakkale ili örneği). Çanakkale Onsekiz Mart Üniversitesi Sosyal Bilimler Enstitüsü, Yayınlanmamış Yüksek Lisans Tezi, Çanakkale.

[25] Jamyang-Tshering, K. (2004). Social competence in preschoolers: an evaluation of the psychometric properties of the preschool Social Skills Rating System (SSRS).

[26] Kazdin, A.E. (1985) Treatment of antisocial behavior in children and adolescents. Homewood, IL: The Dorsey Press.s

[27] Raine, A. (1993). The psychopathology of crime: Criminal behavior as a clinical disorder. San Diego, CA: Academic Press.ss

[28] Tekin M,. Bayraktar G., Y1ldız M., Katkat D. (2006) Beden Eğitimi Öğretmenlerinin Çeşitli Değişkenlere Göre Sosyal Beceri Yeterlilik Düzeylerinin İncelenmesi, Atatürk Üniversitesi Beden Eğitimi ve Spor Bilimleri Dergisi 8(1), 43-59.

[29] Erdoğan E., Sözen H., Cevahircioğlu B.(2014) Farklı Spor Branşındaki Üniversite Öğrencilerinin Sosyal Beceri Düzeylerinin Belirlenmesi. Uluslararası Sosyal Araştırmalar Dergisi, 7 (33) ;828-834. 